\title{
Development of a 3-D Printer and CNC Milling Desktop Machine for Manu- facturing Labs
}

\section{Dr. Jorge Rodriguez P.E., Western Michigan University}

Faculty member in the Department of Engineering Design, Manufacturing, and Management Systems (EDMMS) at Western Michigan University's (WMU). Co-Director of the Center for Integrated Design (CID), and currently the college representative to the President's University-wide Sustainability Committee at WMU. Received his Ph.D. in Mechanical Engineering-Design from University of WisconsinMadison and received an MBA from Rutgers University. His B.S. degree was in Mechanical and Electrical Engineering at Monterrey Tech (ITESM-Monterrey Campus). Teaches courses in CAD/CAE, Mechanical Design, Finite Element Method and Optimization. His interest are in the area of product development, topology optimization, additive manufacturing, sustainable design, and biomechanics.

\section{Dr. Pavel Ikonomov, Western Michigan University}

Associate Professor of Engineering, Design, Manufacturing, and Management Systems, has been working on Virtual Reality simulation and 3D printing for more than 20 years. His main focus has been 3-D modeling design and VR simulation in manufacturing and assembly, medical application, large scale dynamic simulation in various research organizations in Japan like Hokkaido University, TMIT and 3D Incorporated and Virtual Reality Center Yokohama (CTO), UCLA (2001-3) and NIST (2002-3). At NIST he was responsible for industrial Virtual Reality Assembly (VADE) and worked with VR simulation for the optical nano-tweezers. Dr. Ikonomov will oversee the development of 3D printing and VR lab. Dr. Ikonomov has more than 120 journal and refereed conference proceedings publications, three books and book chapter.

\section{Dr. Alamgir A. Choudhury, Western Michigan University}

Alamgir A. Choudhury is an Associate Professor of Engineering Design, Manufacturing and Management Systems at Western Michigan University, Kalamazoo, Michigan. His MS and PhD are in mechanical engineering from NMSU (Las Cruces) and BS in mechanical engineering from BUET (Dhaka). His interest includes computer applications in curriculum, MCAE, mechanics, fluid power, and instrumentation \& control. He is a Registered Professional Engineer in the State of Ohio and affiliated with ASME, ASEE, SME and TAP. 


\section{Development of a 3D Printer and CNC Milling Desktop Machine for Manufacturing Labs}

\section{Abstract}

Nowadays most users of personal machines, or even industrial applications, have specific machine(s) for prototyping and for machining. But the steep increase in demand of machines for personal use, and the fact that those machines provide either one of the two processes, i.e., prototyping and milling, there is a niche market for a machine that can perform both processes. Many consumers, especially in industry, possess manufacturing processes on completely different machines, and few attempt to retrofit one for the other process.

The dual-purpose desktop machine presented here is the result of combining 3D printing and CNC milling, two popular processes, in a useful DIY format for personal manufacturing. With the popularity of people making their own parts at home and the ease of buying components and machines in today's world, even those without experience in these processes can jump in and learn about personal manufacturing.

Important aspects considered were the common components of 3D printing and milling machines, and the crucial acquisition of these components in a form that would allow both processes to work. The machine will be an important learning tool for classrooms and a resource to expose students to manufacturing technologies. It also serves as a level of comparison to available products in the market. Lastly, the machine fits with personal manufacturing by having a smaller footprint, easy operation, and interchangeability of the processes.

Introduction

Additive (material formation) and subtractive (material removal) manufacturing technologies have been popularly employed in a variety of fields such as prototyping modeling artistry, education, decoration, medicine, and direct production. These manufacturing technologies exist as independent machines of large sizes, different structural components, industrial capacities, professional-skill functionalities, and high costs. However, the field of do-it-yourself (DIY) or personal manufacturing is expanding and creating a demand for desktop-sized machines available to the general consumer ${ }^{1}$.

Desktop machines employing subtractive or additive manufacturing processes have become popular for use by hobbyists, but commercially still each process requires a separate machine. In addition, open-source technology has made available hundreds of these newly-emerging machines. But these models also come in a variety of sizes, shapes, capacities, functionalities, and prices; and they are offered by professional companies to DIY enthusiasts spread around the world. The popularity of additive and subtractive technologies, combined with the growth of personal manufacturing, has made three-dimensional printing (3DP) and computer-numericalcontrol (CNC) milling the most desirable technologies for personal use ${ }^{3}$. Therefore, this project sought to combine $\mathrm{CNC}$ milling and 3D printing into one machine; bringing together frame, 
hardware, controls, and software to operate each process. Staying true to the spirit of personal manufacturing, the machine's criteria were defined to have a desktop footprint, an approximate

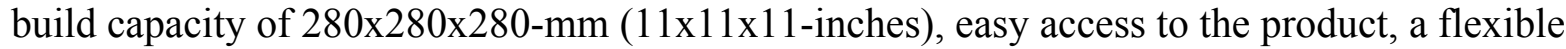
interchangeability of the tools, and a focus on plastic, wood, and soft metals as production materials.

Background

The two processes applied in this work are 3DP and CNC milling. Briefly:

- 3D Printing, a form of additive manufacturing ${ }^{2}$, is the processes of creating a part through the addition of material. 3D printed parts can be made of powder, gels, plastics, and metal. This technology is becoming more common in industry. The most popular type of $3 \mathrm{D}$ printing for personal use is done through fused deposition ${ }^{5}$, which is the melting of plastic through a heated nozzle to lay down layers of material on top of each other to form an object. For the past decade, since around 2005, the 3D printing market has been growing and expanding, which has made available hundreds of personal 3D printers.

- CNC milling is the removal of material through the use of cutters rotating at high speeds. This process is a form of subtractive manufacturing. Materials used for production are generally foam, plastics, wood and metal. These machines are used for prototyping, mass production, mold making and producing custom parts. Manual mills have been used for most of the $20^{\text {th }}$ century, with the advent of computer numerical control coming around the midpoint of the century.

Technically, the motion of both 3DP and CNC milling is controlled through the same programming language. Known as G-code, this programming language is based on the Cartesian coordinate system $(\mathrm{X}, \mathrm{Y}, \mathrm{Z})$, which is used by a variety of other machining and manufacturing processes. This allows implementing other technologies, such as laser engraving and coordinate measuring (CMM), on the same machine. However, research showed that in order to have laser engraving capability, an exhaust system and a casing were needed to get rid of potentially toxic fumes emitted from the laser and burning material, and to enclose the working area, respectively. CMM capabilities were also halted due to the high cost of a single touching probe.

Design Procedure

Basic engineering design process was followed, implying that a design was done based on specifications, and then the manufacturing took place. The machine needed to be strong enough to be able to cut through light metals, and yet precise enough create accurate parts. To accomplish this, the frame was made out of 8020 aluminum extrusions (top, left) due to its great strength-to-weight ratio, ease of assembly, and flexible arrangement ${ }^{4}$. The conversion of rotational motion, from the motors, to linear travel was accomplished by the use of ball screws ${ }^{5}$. The nut of a ball screw contains numerous ball bearings that roll along the threads of the screw, allowing for greatly reduced friction, high precision, and high efficiency. 
For the communication and documentation of ideas, CAD software (Creo Parametric) was used to create and visualize all aspects of the proposed design. The final design of the machine called for four main sub-assemblies (CAD renderings shown in Figure 1): a) base, b) bed, c) bridge, and d) carriage. The base sits flat on the table, containing the ball screw and linear rails for the y-axis motion. The bed is attached to the base, and contains a flat surface for parts to be printed or a work piece to be secured.
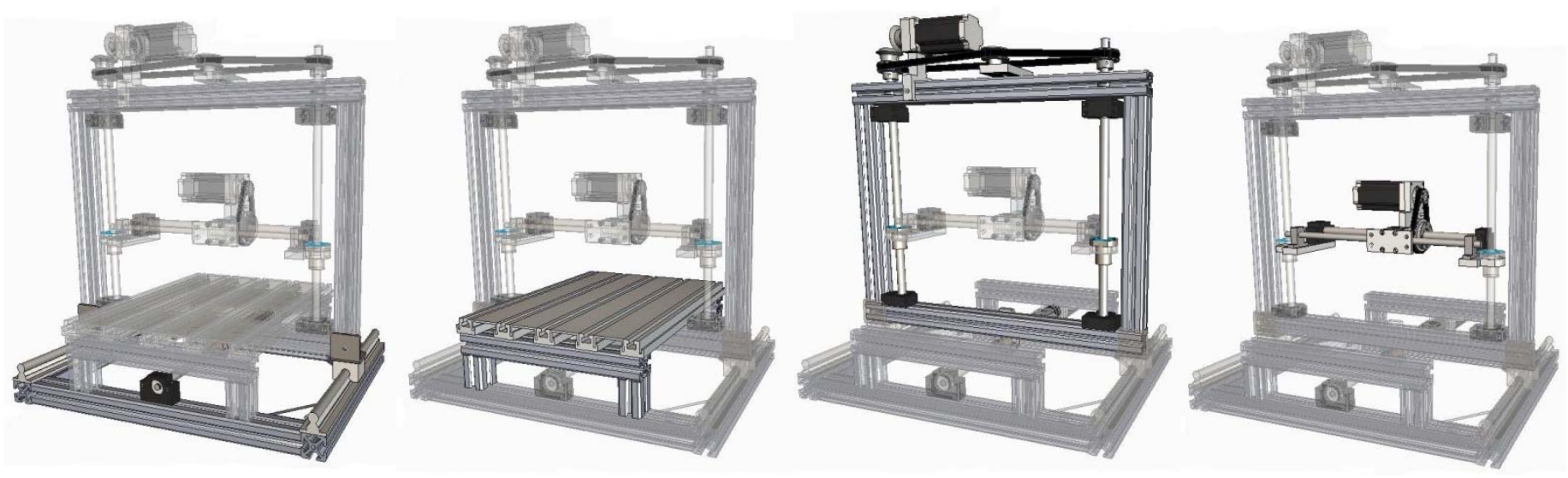

Figure 1. CAD rendering of the four main sub-assemblies: a) base, b) bed, c) bridge, and d) carriage.

The bridge is attached to the y-axis ball screw and rides along the linear rail of the base ${ }^{6}$. It consists of the two ball screws for the z-axis motion, which lifts the ball screw and linear rail of the x-axis up and down. On top of the bridge, the motor for the z-axis lies perpendicular to the ball screw, thus a pair of bevel gears are used to translate the motion from the motor to the screw. A pair of pulleys and a belt connects the two z-axis ball screws, keeping them synchronized with each other and maintaining the $\mathrm{x}$-axis level.

The carriage is the assembly that travels along the $\mathrm{x}$-axis and holds the tool for the desired process. While the $\mathrm{x}$-axis ball screw is fixed, the ball nut is allowed to rotate to generate the linear travel. Consequently, the motor for the carriage moves along with the entire assembly. This is accomplished by a custom bracket that not only holds the motor in place, but also contains a bearing in which the ball nut rotates. The motor and ball nut are connected by a beltand-pulley system, which is held attached to the ball nut with a bushing. The interchangeable tool system uses a matching-hole pattern for both the spindle and the extruder head brackets, each of which can be fastened with the same group of bolts.

Fabrication/Assembly Procedure

After the design was complete, the aluminum extrusions were cut to length on a band saw. One piece of aluminum extrusion needed to have two one-inch holes cut through the middle, using a drill press, to get a coupling inserted through the holes. Four bed supports were cut and milled to ensure that the work piece was on a level surface. 
A total of fifteen brackets needed to be made for the machine (Figure 2). These brackets were used to mount the stepper motors, to connect the different axes of the machine, to connect couplings for the motors to the ball screws, and to allow tooling to be interchangeable. The brackets were made from 6061 aluminum alloy, which is the same type of aluminum as the frame, keeping the machine strong and light weight. All of these parts were made in the student machine shop where a manual mill and a manual lathe were utilized.

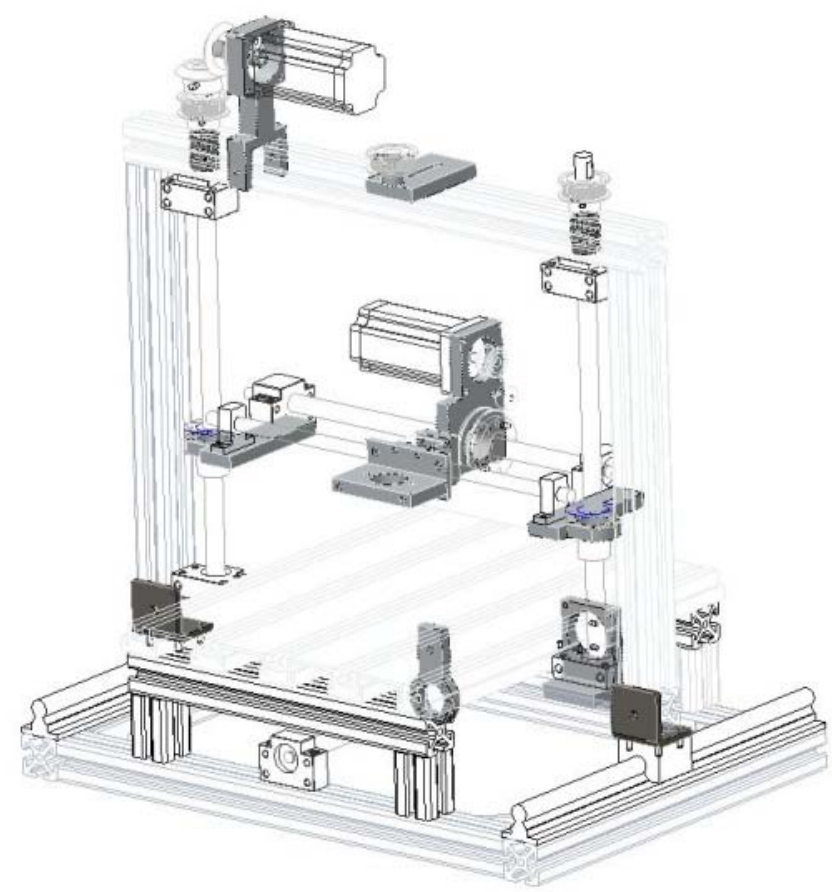

Figure 2. Main components for mounting of components, in CAD.

Alignment issues were addressed during the assembly, specifically when the motion control components were attached to the frame. On the base, the y-axis ball screw and the two linear rods were aligned parallel to one another to prevent binding of moving components and to prevent damage. Parallel alignment was achieved by using large gauge blocks between the ball screw brackets and the edge of the linear rods, making sure they were equidistant at both ends.

The z-axis has two ball screws that run up and down the bridge, holding the components for the $\mathrm{x}$-axis and carriage. With two ball nuts, which also had to be equidistant on both ends, this was achieved with gauge blocks placed at the top and bottom between the nut and the ball screw supports. The final assembly was done on the carriage. To make sure that this was level, the brackets attached to the z-axis ball screw nuts had to be level to the bed. Finally, the x-axis brackets were aligned parallel and placed at the same distance from the ball screw bracket with gauge blocks once again. 


\section{Electronics and Software}

The full frame motion for both processes was controlled by the same electronic components, while the individual tools for each process had their own manual controls ${ }^{4}$. The heart of the electronics is the breakout board, which is the intermediary between the tool-path-generating software and the motors that provide motion. The board was powered by its own 24-volt power supply, allowing the board to communicate with the software, interpret the program, and send out different electrical signals.

Connected to the board were the micro-stepping drivers, which relay the pulses and signal directions from the board to the motors. The drivers and the motor motions were powered through a 48-volt power supply. The milling spindle head (Figure 3a) was powered by a second 48-volt power supply. The amount of power supplied controls the revolutions per minute (rpm) of the spindle. With a pulse-width modulator between the spindle and the power supply, the power and the rpm are controlled manually.
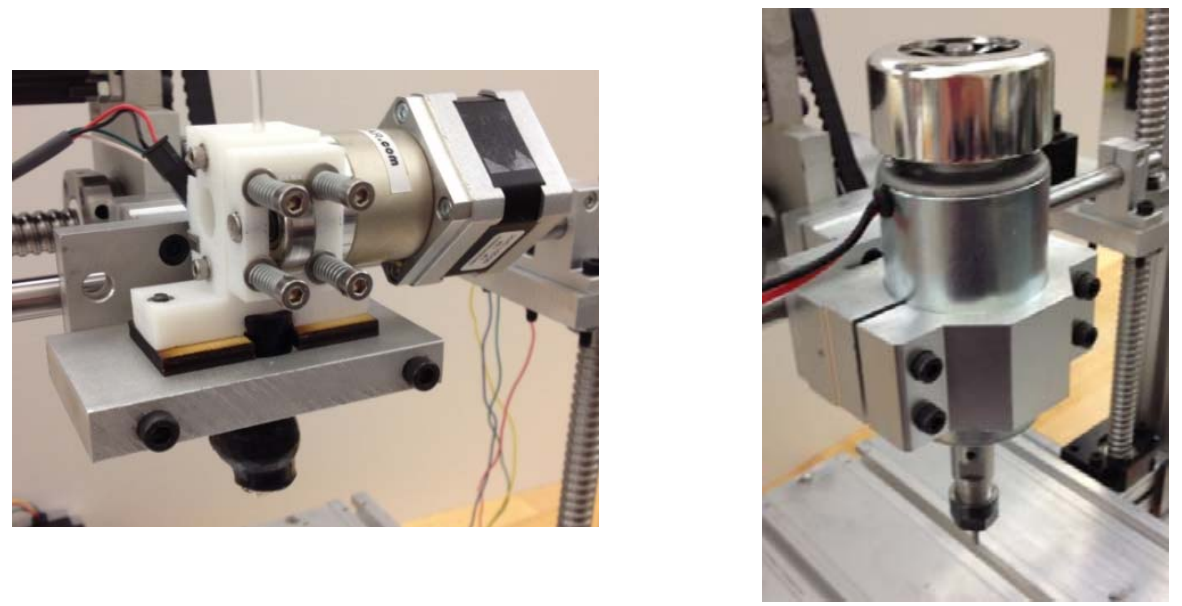

Figure 3. Photographs of processing heads: a) 3D printing, b) milling.

The 3D printing head (Figure 3b) has two main components: a stepper motor, which drives a gear system that feeds the plastic through the nozzle, and a heating element. The motion of the stepper motor is directed by another driver connected to the board and powered by the first 48volt power supply. The heating element is powered by the second 48-volt power supply used for the spindle, but a proportional integral derivative controller is connected between the two. This controller powers and sets the required temperature of the nozzle to melt plastic filament. The temperature controller uses mathematical equations that calculate the error from the feedback of a thermistor to keep the temperature constant.

The software used for the machine is called USBCNC. This program gets its namesake from the fact that it allows communication between a computer and the board through a USB port, as opposed to the usual parallel port interface. The software is able to use Autodesk part files to create a tool path. Image files and completed programs from computer-aided manufacturing 
(CAM) software can also be imported into the software ${ }^{7}$. Since the speed of the spindle and the temperature of the extruder are controlled manually, the software uses the same type of G-code programming for the motion of both processes. The 3D printing tool path (Figure 4), however, must be generated from an STL file of a CAD model, which gets sliced and programmed through a software called Slic3r. The G-code obtained can be imported into USBCNC.

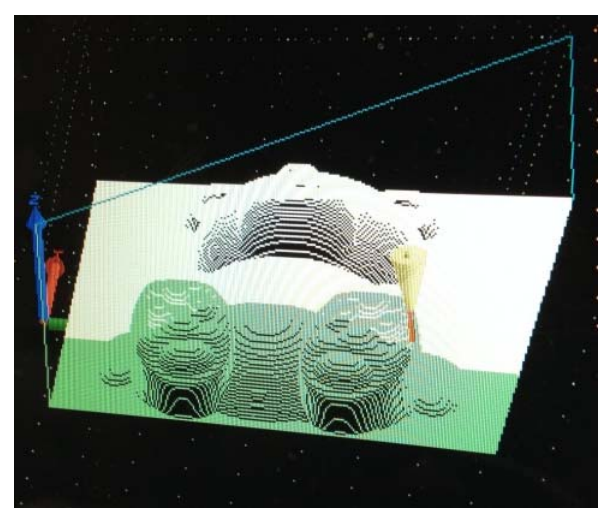

Figure 4. Tool path simulation in software..

Results and Discussion

The machine's frame footprint was 61-mm (2-ft) squared. Along with a mounting board for the electronics, the total footprint increased to $92-\mathrm{mm}$ (3-ft) squared. The build envelope was approximately $280 \times 280 \times 280-\mathrm{mm}(11 \times 11 \times 11$-in) and with the addition of limit switches to keep

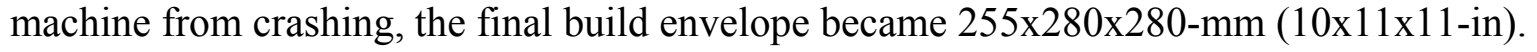
Nevertheless, the build envelope is open and easy access to the work piece comes from both the front and back of the machine.

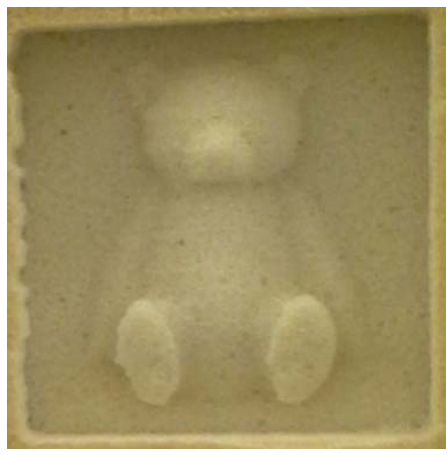

Figure 5. Result from test of CNC milling.

Milling capabilities were first tested by machining parts out of foam. The machine was able to create a 3D image of a bear in the foam (Figure 5), along with a variety of 2D engravings to test the accuracy of the machine. Tolerances of less than $1 \mathrm{~mm}(0.04 \mathrm{in})$ were achieved. These same results were reproduced in wood as well. Some simple two-dimensional engravings were made in metal, but further capability testing is needed. 
The 3D printing process was tested, deposits of plastic were made, but these needed the use of a heated bed and fine-tuning of the extruder settings to get better results. It was not possible to complete a full part before a driver malfunction, making 3D printing inoperable until another driver is delivered. Despite the obstacles, the entire 3D printing motion and the extruder motor worked effectively with USBCNC.

Each of the tooling is connected by a set of nuts and bolts with the same hole-pattern onto a bracket, allowing each process to be changed as desired. The functionality of each process is ensured by properly connecting the electronics to their respective power supplies, and by setting up the manual controls. In USBCNC, the fourth axis of motion must be either enabled for 3D printing or disabled for milling. At this point, a program can be created and run.

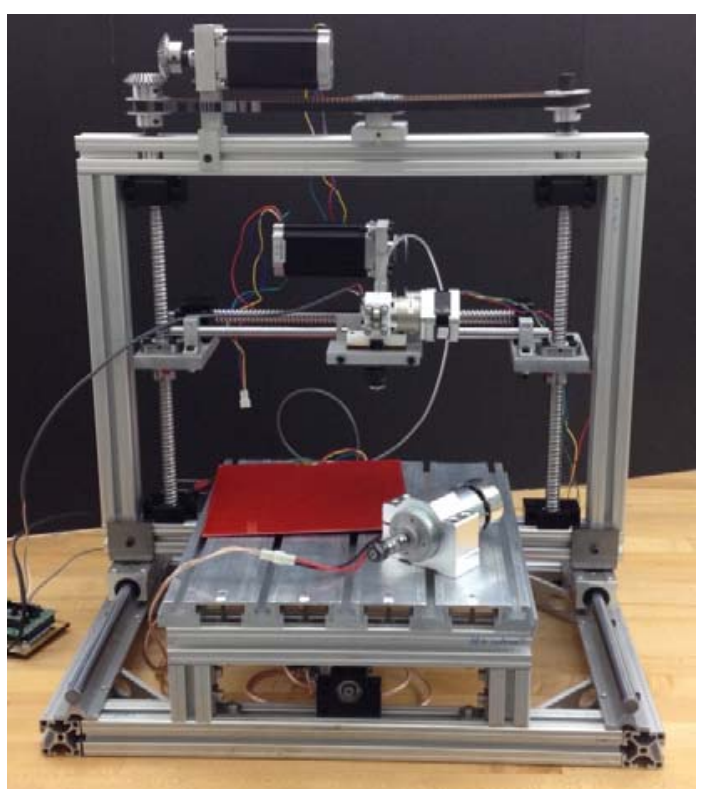

Figure 6. Photograph of final dual-purpose desktop machine.

\section{Conclusions}

This project dealt with the creation of a machine capable of two different types of manufacturing processes by utilizing the common components and reducing the space needed for each independent process. The successful completion of this project adds to the DIY community additional options to improve the functionality and the ability to create parts. The market is wide open for machines that can do multiple processes as people gain more access to these technologies.

The design criteria and objectives for this project were met. The machine has a desktop-sized footprint and can easily have space to be used in any home, while having an approximate 
255x280x280-mm (10x11x11-in) working envelope and easy access to the product. The machine can also be placed onto a smaller desk if the electronics are moved to the floor.

With the ability to produce parts that are within a tolerance of $1 \mathrm{~mm}$ and with a final cost under $\$ 1,500$, though cost was not a primary design criterion, the specifications of this dualpurpose desktop machine is a valid option to one-process machines on the market; but having the advantage of an easy changeover procedure that can switch between 3DP and milling in a matter of minutes.

This was a student project that provided an excellent opportunity for the mechanical design students to expand their comfort zone, mainly into the electronics area. It was very challenging at times, but at the end they did finish the project. The desktop machine is currently used as a demo machine in our manufacturing labs.

Acknowledgements

The dual-purpose desktop machine was made possible thanks to the guidance from Peter Thannhauser and Abraham Poot for their assistance in electronics, to Glenn Hall and Mike Konkel for their help in the machine shop, and the direct participation of Austin Grieve, Kenneth Klingler, and Miguel Acuna. Finally, our gratitude goes out to the Office of the Vice President for Research (Western Michigan University) and to Misumi USA for their financial assistance.

Bibliography

1. Anderson, C. (2012). Makers: The new industrial revolution. N.p.: Crown Business.

2. ASTM Standard F2792-12a (2009). Standard terminology for additive manufacturing technologies. ASTM International, West Conshohocken, PA, 2003, DOI: 10.1520/F2792-12A.

3. Bradshaw, S., Bowyer, A. \& Haufe, P. (2010). The intellectual property implications of low-cost 3D printing. 7:1 SCRIPTed 5, http://www.law.ed.ac.uk/ahrc/script-ed/vol7-1/bradshaw.asp.

4. Breaz, R., Racz, G., Bologa, O., and Oleksik, V. (2012). Motion Control of medium size CNC Machine-tools A hands on approach. $20127^{\text {th }}$ IEEE Conference on Industrial Electronics and Applications. ICIEA 2012, pp 2112-2117.

5. Devijver, S. (2011). Building your own 3D printer. In RepRap. Retrieved March 19, 2013, from http://reprapbook.appspot.com/\#d0e148.

6. Patrick Scott (2005). The straight story on ball screws. Motion System Design. Vol. 47, Issue 12, pp 19.

7. X. Xu, H. Wang, J. Mao, S. Newman, T. Kramer, F. Proctor, J. Michaloski (2005). STEP-compliant NC research: The search for intelligent $\mathrm{CAD} / \mathrm{CAPP} / \mathrm{CAM} / \mathrm{CNC}$ integration. International Journal of Production Research, Vol. 43, Issue 17, pp 3703-3743. 